\title{
Low Cost Data Acquisition System for Condition Assessment of Electric Motors
}

\author{
Yassen Gorbounov $^{1}$, Tihomir Dzhikov ${ }^{2}$ \\ Associate Professor, Dept of Mining Automation, University of Mining and Geology "St. Ivan Rilski”, Sofia, Bulgaria ${ }^{1}$ \\ PhD Student, Dept of Mining Automation, University of Mining and Geology “St. Ivan Rilski”, Sofia, Bulgaria ${ }^{2}$
}

\begin{abstract}
The requirements for the effective control, safe and reliable operation of electrical motors and drives are constantly growing. Therefore an accepted maintenance strategy for the detection of nascent faults and for avoiding unexpected failures is the condition monitoring of their health status. This allows to perform preventive diagnostics and a planned repair instead of emergency one. This paper represents an ongoing research on elaborating a low cost data acquisition system for condition assessment of electric motors based on the typical profile of its current, voltage or vibration. This approach is typically non-invasive and is known as a signature analysis. Some typical fault conditions are systematized in the paper and some perspective methods for monitoring and diagnosis of faults in electric motors are discussed. An approach towards building such a system using low cost hardware and open source software is proposed.
\end{abstract}

Keywords: Condition Monitoring, Motor Faults, Signature Analysis, Fault Diagnosis

\section{INTRODUCTION}

Electric motors are major consumers of electrical power on a global scale as they are involved in all areas of life medicine, industry, household appliances, and so on. According to various sources [1] [2] [3], they consume an average of more than $50 \%$ of the total electricity produced. This gives them a special place in drive systems and therefore, the demands for their control and reliability are constantly growing. This fact leads to the research and development of more and more complex and faster control algorithms and advanced maintenance techniques at the same time. However, any machine is subject to failure that can deteriorate its normal operation. Different kinds of failures can originate in both the electrical subsystem and in the mechanical subsystem of the motor or the drive. The earlier the faults are detected, the smaller the material and financial losses. Performing preventive diagnostics is very important as it allows the substitution of emergency repairs with planned ones and can have a serious economic effect.

Various condition-based maintenance schemes are in general used together with conventional planed maintenance strategies [4]. There already exist well developed methods to observe definite state variables and further compare them or their predicted values with reference values in order to discern the healthy machine state from erroneous operation and even to differentiate distinct types of failures. These methods are usually mathematically complex and require significant computational power. Commercial solutions are available on the market but their cost can reach a magnitude of tens of thousands of US dollars [5] [6] and that prevents them from being widely used.

The burst development of digital electronics and the heightened computing power together with the increasing number of open-source solutions makes it possible to collect data and to develop complex mathematical algorithms for digital processing at significantly lower price. This makes in turn the automated fault detection and analysis methods accessible by a wider audience of engineers and applicable for lower power motors. This paper makes a brief review of the more common faults in electric motors. Some perspective methods for analyzing faults, such as ways of performing signature analysis, are discussed. An ongoing research on elaborating a low cost data acquisition system for condition assessment of electric motors is introduced together with a proposal for further development of the system.

\section{TYPICAL FAULT CONDITIONS IN THE ELECTRIC MOTOR DRIVE SYSTEM}

A typical motor drive system consists of the components depicted in Fig. 1. A facility electric power distribution system is comprised of wires and transformers and it provides the power for the inverter and the motor. The motor itself is driven either directly by the power distribution system or by the adjustable drive speed inverter. Next the motor shaft is coupled mechanically to the load by the aid of belts or other power transmission devices. The mechanical load refers to the process-specific equipment such as a fan, a pump, a conveyor or some other device. And every node in this configuration is mounted on a pedestal. Each of the above components tends to fail at a certain stage in its life for various reasons. Some of the common potential places and reasons are shown with numbers in the figure. For instance 


\title{
International Advanced Research Journal in Science, Engineering and Technology
}

\author{
Vol. 5, Issue 10, October 2018
}

the mechanical mounting of the motor and the load onto a pedestal (1) can cause augmented vibration due to an angular or radial misalignment that can emerge during the installation or the exploitation stage. The root cause of failures in the electrical motor (2) may be provoked by one or a combination of the following factors: broken bearings or problems with their lubrication, contamination which leads to excessive heat generation, broken rotor bar of an induction motor, insulation rupture, wire displacement or short in a wire, eccentricity or mechanical imbalance, loose electrical contact, induced shaft currents etc.

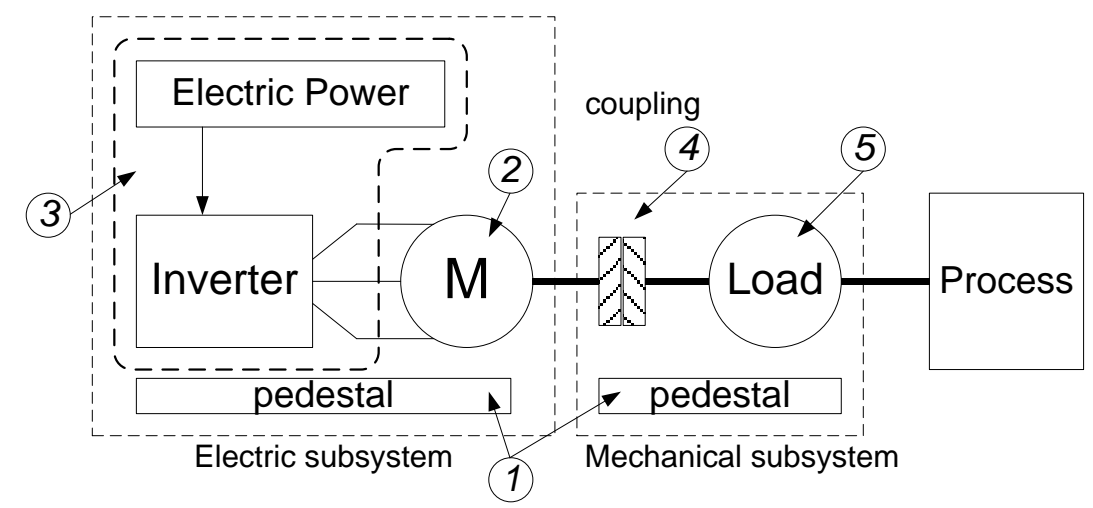

Fig. 1 Generalized overview of the electric motor drive system

The electric power supply system (3) can induce faults by incorrectly driving the motor due to a missing phase, voltage or current imbalance, overvoltage or undervoltage, overload, negative current sequence, some components wear (such as a capacitor) etc. All these reasons inevitably modify the harmonic spectrum of the power supply of the motor. The function of a coupling (4) is to connect two rotating shafts for the transfer of rotary motion and torque. The problems caused by the coupling are mainly related with factors such as excessive misalignment, improper installation which causes torsional vibrations, wrong amount of dampening, lack of torsional rigidity or backlash, ruptured or cracked element. The load (5) can also impact the motor operation by radial misalignment, axial thrusting, increased belt tension or inappropriate amount of mechanical loading. More than $66 \%$ of the failures overall are detected during operation and about $28 \%$ are detected during maintenance procedures [7]. As it can be seen some of the failures are related with the electrical subsystem while others are related with the mechanical subsystem. A non-exhaustive summary of the typical failures is given in Fig. 2 [8] [9] [10] (with additions).

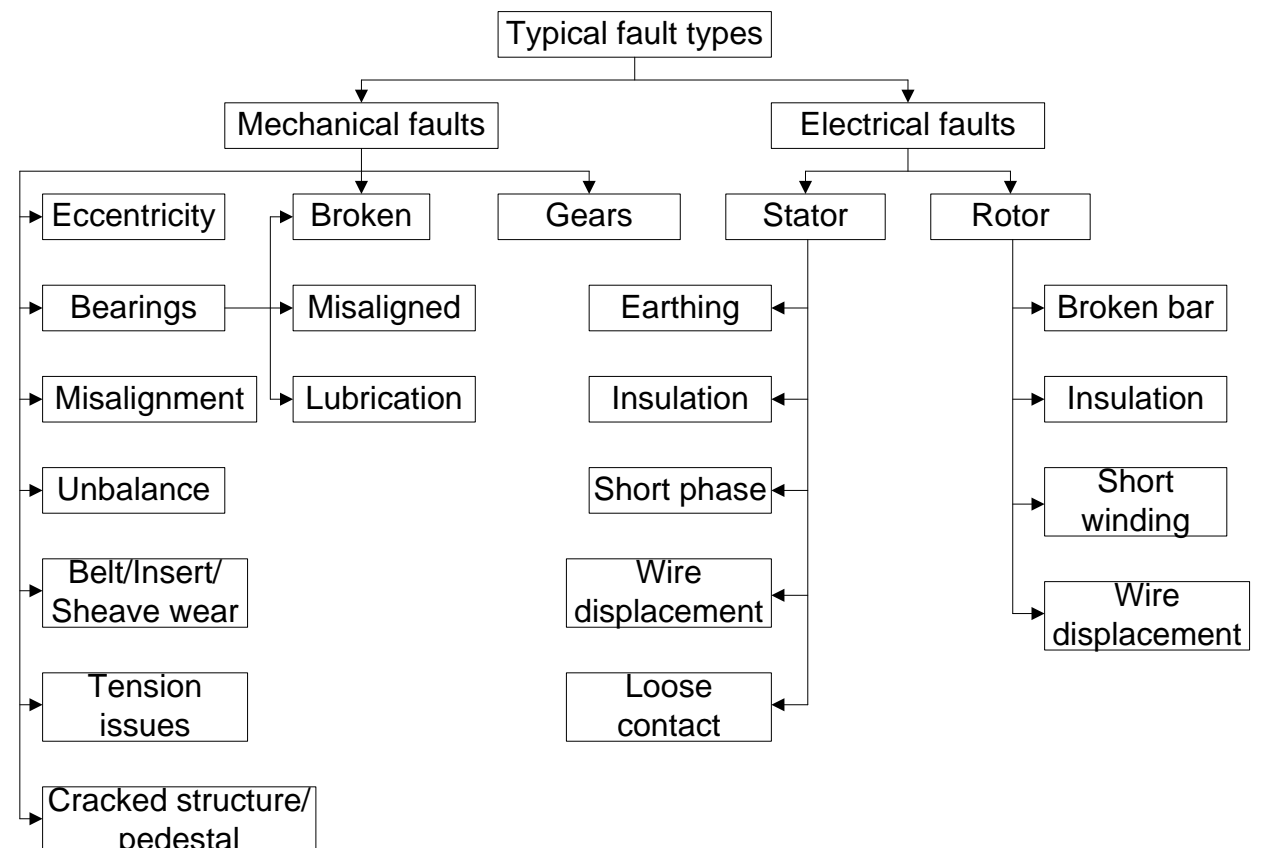

Fig. 2 Classification of the most common causes of damage to electric motors

All of the above reasons have a sensible impact on the vibration and the current of the motor which can be measured and further processed. Informative features can be extracted by observing the signature profile of the motor. Rotor 


\title{
International Advanced Research Journal in Science, Engineering and Technology
}

\author{
Vol. 5, Issue 10, October 2018
}

faults can be observed either through monitoring the rotor currents, if there are any windings on the rotor at all, or more frequently by monitoring the stator currents.

\section{CONDITION MONITORING METHODS}

The condition monitoring methods represent the signal analysis stage and take place immediately after measuring and conditioning the vibration, the torque or the current of the motor. They can be separated into diagnostic methods and subsequent decision techniques and are summarized in Fig. 3.

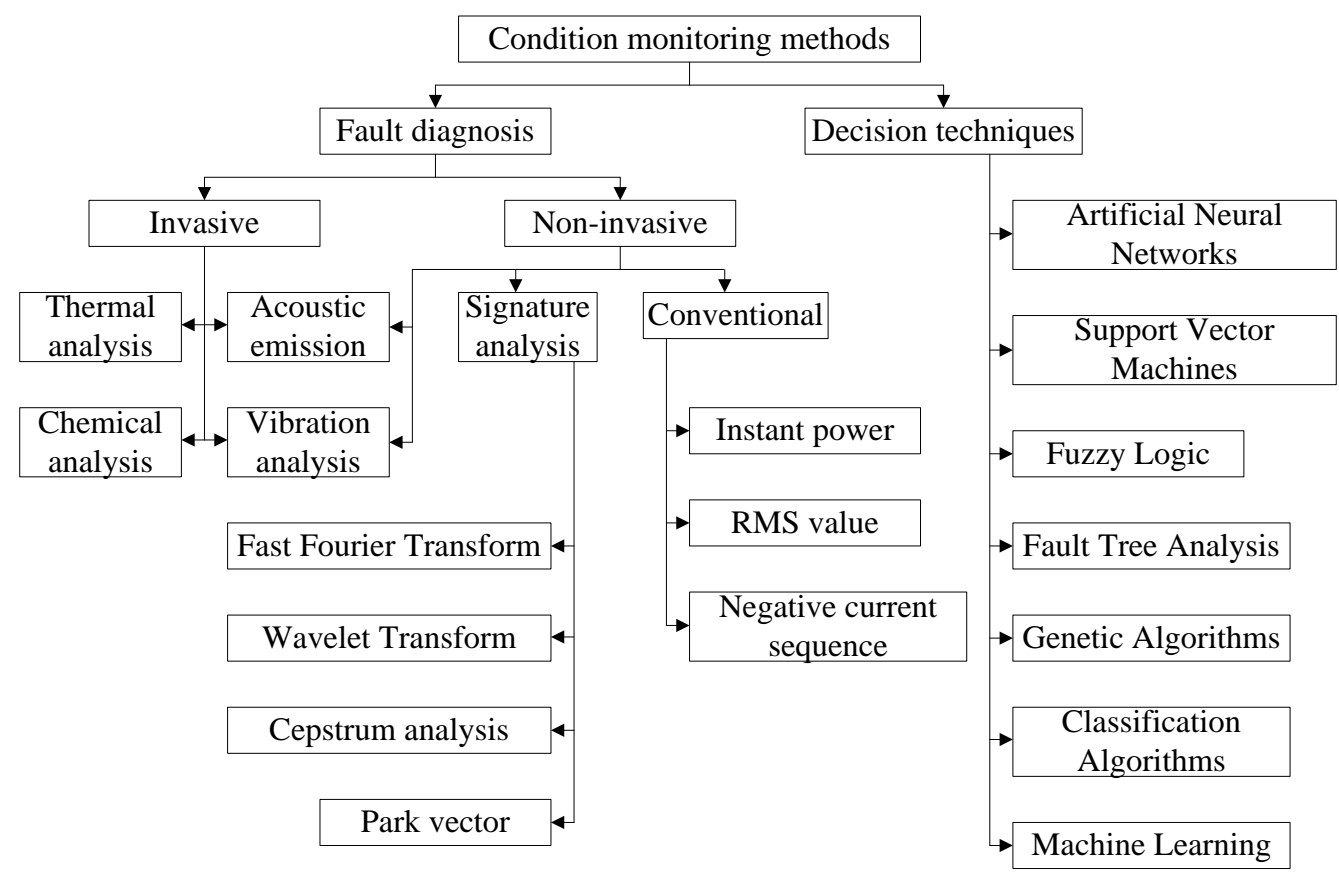

Fig. 3 Condition monitoring methods and decision making techniques

The fault diagnosis includes both invasive and non-invasive approaches with the latter being preferred over the former. The thermal and chemical analysis requires sensors to be mounted inside the motor body often needing a modification in the mechanical construction. In the same time this type of analysis is not informative enough since it does not provide signature profile data. The acoustic emission and the vibration analysis on the other hand may be classified as both invasive and non-invasive methods depending on the way they are being used. In general the group of noninvasive methods can be subdivided into conventional and signature analysis categories. The conventional methods include instant power, RMS value and negative current sequence (NCS) monitoring which provides limited amount of information and is not very suitable for predictive maintenance. The RMS method represents a simple statistical calculation and is mainly used for a rough indication of the motor loading. Stator fault detection is largely based on the NCS which is null for healthy motors powered by symmetrical multiphase voltage. If a turn-to-turn fault emerges, it breaks the symmetry and leads to negative sequence current which provides an indication of fault presence and in some sense it can be used as fault severity measure. The NCS method is often employed for detecting winding faults and for the detection of eccentricity symptoms in the motor current.

The most advanced and prospective methods lie in the group of signature analysis which are non-invasive [13] thus providing capabilities for evaluating a remote condition assessment of electric motors. They can be subdivided into three major categories namely vibration analysis (VA) [8] [12], motor current signature analysis (MCSA) [14] [15] and load torque signature analysis (LTSA) [11].

The time-frequency domain analysis is using a representation of the Fourier decomposition, called the Fast Fourier Transform (FFT). Many mechanical and electrical failures generate signals whose frequencies can be determined by knowledge of motor parameters. These fault signals can appear in vibration, current and flux signals. The FFT analysis is based on the fact that each periodic signal can be decomposed into a sum of sinusoids thus converting the signal from the time domain into the frequency domain. In [16] FFT is being used to detect real-time asynchronous motor damage broken rotor rod, bearing failure, eccentricity and short circuit. In contrast to the FFT the wavelet transform (WT) decomposes the signal into a set of non-sinusoidal waves. It is usually applied to impulse waveforms that are not convenient to be represented as a sum of sinusoidal components. This transform is being initially used for seismic 


\title{
International Advanced Research Journal in Science, Engineering and Technology
}

\author{
Vol. 5, Issue 10, October 2018
}

analysis. It provides capabilities to extract local parameters of the signal with very good precision. In [17] the WT method is being used for broken rotor bar fault detection in induction motors. The Cepstrum analysis function is initially developed for characterizing the seismic echoes resulting from earthquakes and bomb explosions. It is also used to determine the fundamental frequency of human speech, to analyse radar signal returns, and to evaluate machinery vibration. It is a result of taking the Inverse Fourier Transform (IFT) of the logarithm of the estimated spectrum of a signal. The word "cepstrum" is derived by exchanging the two halves of the word "spectrum". In [18] the cepstrum is described as "the power spectrum of the logarithm of the power spectrum". Operations on cepstra are labeled quefrency analysis, liftering, or cepstral analysis. In [19] and [20] Cepstrum analysis is being used for bearing fault detection. The Park vector method is a relatively new technique, which is being applied in the diagnosis of rotor faults detection, inter turn stator faults and unbalanced supply voltage detection [21]. An undamaged machine theoretically shows a perfect circle when plotting the two axes namely the active stator current and the reactive stator current $(\mathrm{d}, \mathrm{q})$ obtained from the Park transform. An unbalance due to turn faults may result in more elliptic representation of the plot giving a clear indication of motor fault.

After diagnosing a potential fault condition it may be classified by an expert. The state-of-the-art of computing devices gives opportunity to automate this final step and obtain fault information as early as possible and with a minimal error. In Fig. 3 several decision techniques are shown. The topic is quite broad and many research work has been done worldwide so it is beyond of the scope of this paper. It should be noted only that in general these techniques can be subdivided into classification algorithms such as the Support Vector Machines (SVM) and intelligent algorithms such as the Machine Learning (ML) and Artificial Neural Networks (ANN) which seem to be the most perspective.

\section{THE EXPERIMENTAL DATA ACQUISITION SYSTEM}

The general overview of the experimental setup is presented in Fig. 4. It consists of a motor and a mechanical load mounted on a pedestal in such a way that permits a radial or angular misalignment or other type of imbalance to be introduced. The main sensing components being used in motor failure analysis include accelerometers, Hall sensors, Rogowski coils and current transformers. That is why some of them exist in the experimental setup. There exist various types of accelerometers such as Piezoelectric, Capacitive Micro Electro Mechanical System (MEMS) and Piezoresistive [22]. A low cost capacitive MEMS type is used here for vibration analysis. It is the ADXL335 3-axis accelerometer by Analog Devices which has a bandwidth of $1600 \mathrm{~Hz}$ for the $\mathrm{X}$ and $\mathrm{Y}$-axes and $550 \mathrm{~Hz}$ for the $\mathrm{Z}$ axis and this is sufficient for most experiments. The Rogowski coil and current transformers are very precise and flexible to mount solutions but they come at a price. That is why a Hall-effect-based linear current sensor such as the ACS758 is appropriate here. It can withstand AC currents of $100 \mathrm{~A}$ because of its very low internal shunt resistance of the order of 100 micro ohms.

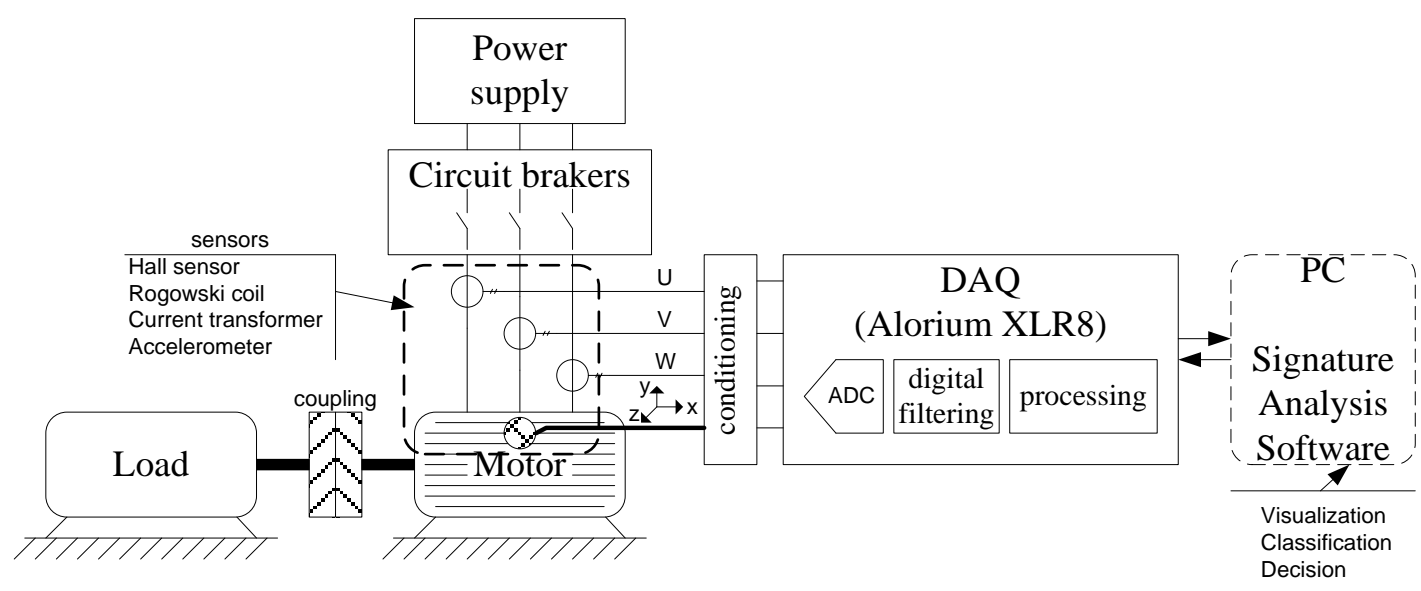

Fig. 4 Experimental setup with the data acquisition system

Both vibration and current monitoring techniques require conversion of the measured quantity into voltage and subsequent fast data acquisition. This process imposes signal conditioning and filtering in order to limit the spectrum of the signal, subsequent transformation and scaling/normalization, data reduction and sampling. Since the data processing path can be too long and thus significantly slow, more and more common nowadays become programmable logic devices such as the Field Programmable Gate Arrays (FPGA). Because these integrated circuits don't have hard logic architecture (like ALU, registers, etc.) but only logic gates and memory, they are capable of implementing parallel running algorithms. It makes it possible to embed the signal conditioning, analog to digital conversion, signal decomposition (FFT or WT) and an artificial neural network onto a single device. This way a true real time data 


\title{
International Advanced Research Journal in Science, Engineering and Technology
}

\author{
Vol. 5, Issue 10, October 2018
}

processing and decision making can be done. Existing products on the market are expensive and quite prone to negative effects of harsh environment for continuous condition monitoring. Instead, simple, low cost, robust and physically small solutions constitute a significant need in the market today. Such a platform is provided by Alorium Technology [23] by their XLR8 Arduino-like platform - see Fig. 5 (a). The core of this board is the Intel (formerly Altera) MAX 10 FPGA device. It has an integrated 12-bit successive approximation register (SAR) type analog to digital converter (ADC) with up to 17 inputs and a cumulative speed of up to 1 million samples per second (MSPS). The MAX10 FPGA is capable of handling both digital and analog types of sensors. In addition it has one 18-bit $\mathrm{x} 18$-bit embedded multiplier (divisible into up to two 9-bit x 9-bit independent multipliers). The XLR8 board has an embedded 8-bit AVR instruction set compatible soft-core IP microcontroller together with a free reconfigurable FPGA logic gates which is shown in Fig. 5 (b). As it can be seen the AVR soft-core MCU coexists together with the so called accelerator blocks (XB). Each XB is an optimized hardware implementation of a unique processor intensive function and new XBs can be designed by the end user in the Intel Quartus IDE environment. Although currently all the data acquisition is done in software these XBs can offer significant acceleration by running critical algorithms such as Park vector and even an ANN entirely in hardware.

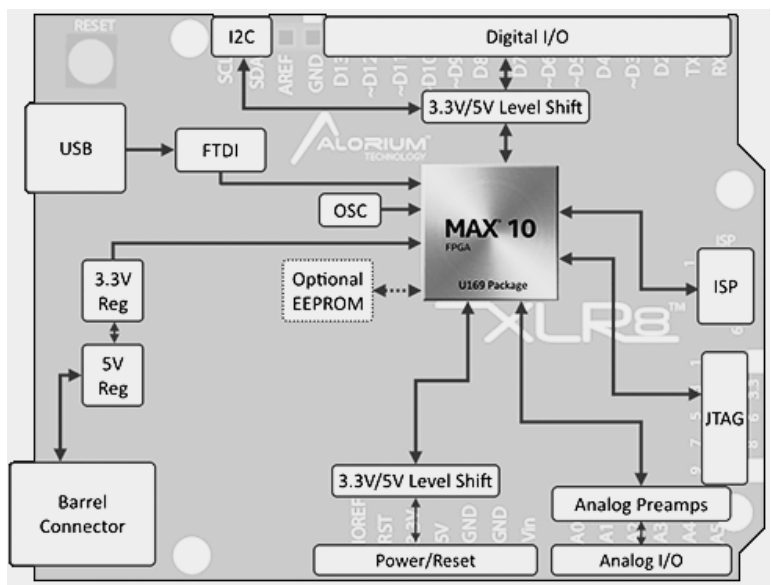

(a)

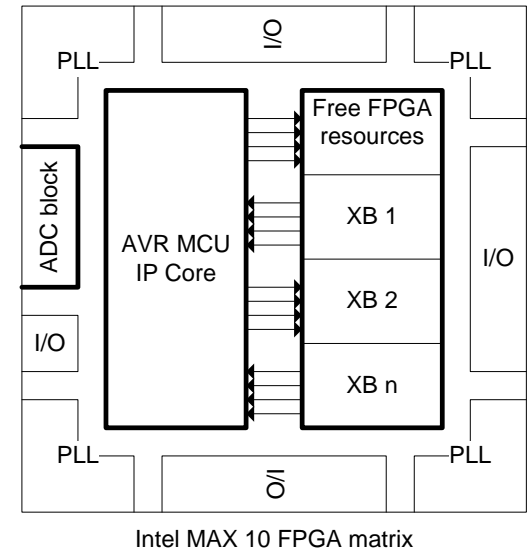

(b)

Fig. 5 Alorium Technology XLR8 board (a) and Intel MAX 10 FPGA configuration by Alorium (b)

The data analysis is usually performed in software either by proprietary vendor software that is available with the specialized measurement equipment or by professional mathematical packages such as Matlab that are commercially available. Although quite powerful these solutions are not very flexible because of their closed-source architecture and in some cases they can be quite expensive. There exist also software alternatives that are powerful enough and can be freely downloaded. One such Matlab alternative is the Scilab [24] mathematical package which has a very similar syntax. Add-on packages such as NumPy, SciPy, and Matplotlib make the Python programming language an attractive alternative to Matlab, and there is an emerging trend for some Matlab users to switch to Python. As a proof of concept the solution given in [25] has been evaluated here in order to do vibration analysis. It is a free program written in Python and intended for machinery vibration analysis in the time and in the frequency domain. A sample result from a vibration measurement of an induction motor running at $800 \mathrm{rpm}$ is given in Fig. 6.
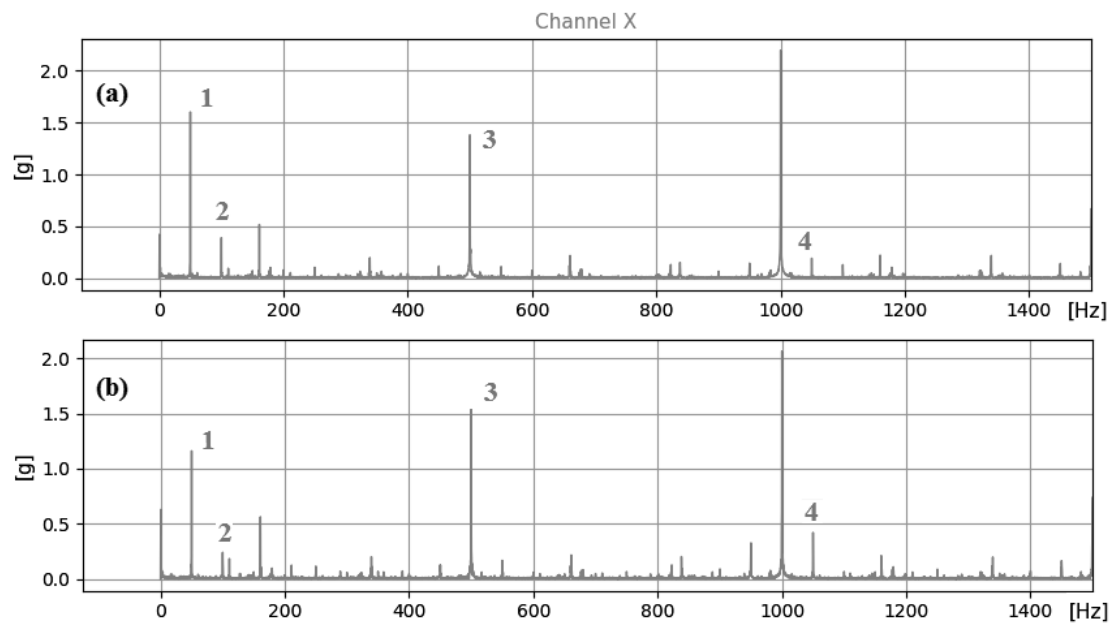


\section{International Advanced Research Journal in Science, Engineering and Technology}

Vol. 5, Issue 10, October 2018

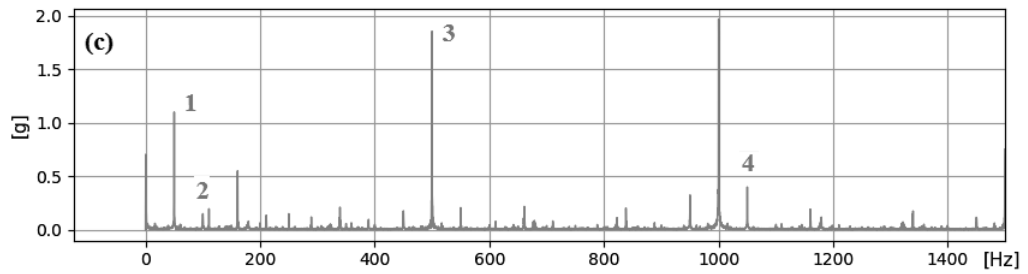

Fig. 6 Vibration harmonic contents for an induction motor running at $800 \mathrm{rpm}$ under normal operation (a), angular misalignment (b) and radial misalignment (c)

The figure represent the harmonic spectrum of the vibration of the motor when it rotates under normal operation conditions (Fig. 6 (a)) or with introduced angular (b) or radial misalignment (c). With numbers in the figure they are pointed out the observed differences which can be further classified.

\section{FUTURE WORK}

As it was mentioned earlier FPGA devices have inherent capabilities of implementing parallel running algorithms. Together with the embedded microcontroller the XLR8 board provides an excellent platform for building a single system-on-a-chip low cost data acquisition system for condition assessment of electric motors. The future development of the proposed platform requires a systematic approach. The first stage includes the design of modules to extend the software given in [25] to perform other analysis tasks such as Cepstral analysis or Park vector analysis. Helpful and freely available resources are given in [8] and [26]. The second stage includes the design of new XB blocks that can perform the above algorithms in hardware and thus to implement a real-time signature analysis. A general overview of the complete data acquisition system for condition assessment of electric motors is given in Fig. 7.

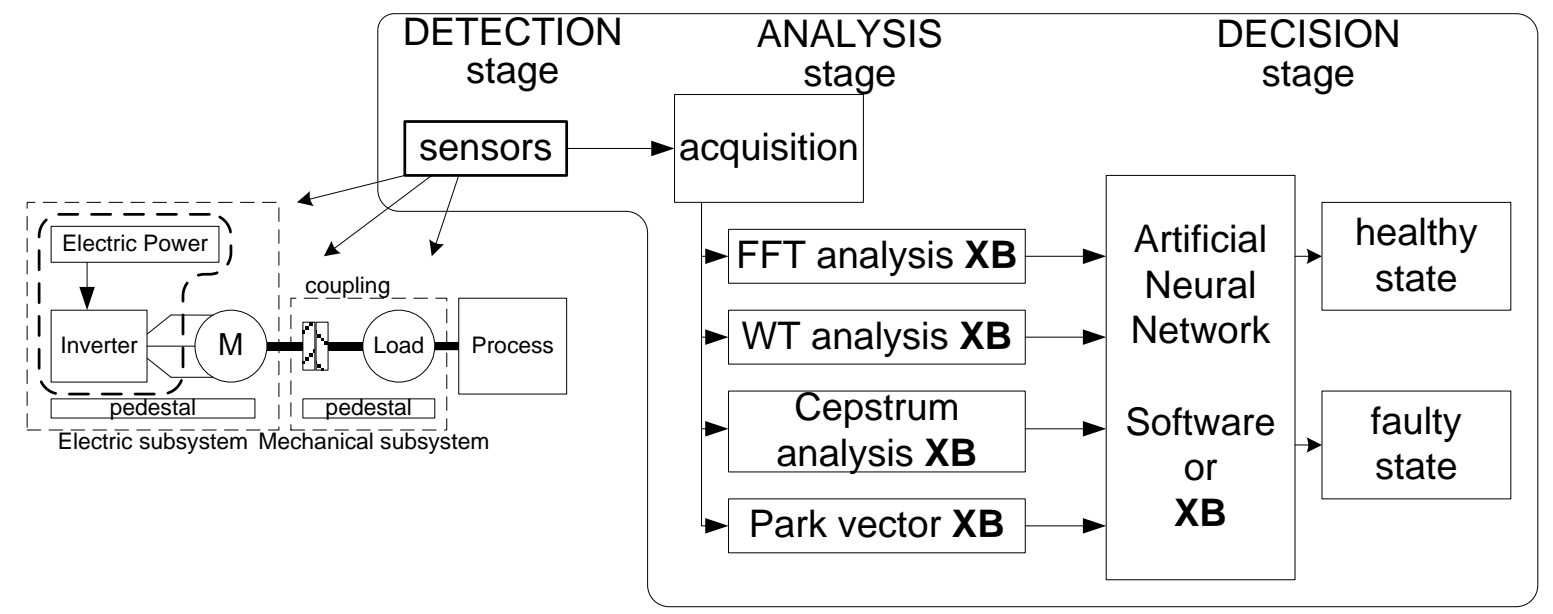

Fig. 7 Generalized overview of the low-cost data acquisition system for condition assessment of electric motors

\section{CONCLUSION}

There exist a number of diagnostic methods for identifying different failure situations of electric motors such as mechanical vibration monitoring, thermal imaging, acoustic monitoring, etc., but most of these methods require expensive sensors or physical interference in the construction of the electric motors. It is known that the various kinds of failures influence the shape of the voltage, the current, the vibration or the torque. This allows it to be analyzed in a sensorless and non-invasive fashion which is done mainly by performing signature analysis. The analysis of the signal profile is done by observing its harmonic spectrum and searching for typical frequencies (artifacts. In case of a fault, higher harmonics emerge that are not typical for the healthy machine. This method allows for early detection of failures and helps transforming the maintenance process from reactive to preventive to predictive one. This makes the topics discussed in the paper relevant, practical, and with good potential economic impact. Commercial solutions are available on the market that can perform condition assessment of electrical motors but their high price limits their usage. A lowcost alternative has been discussed in the paper. It employs the utmost performance of the FPGAs provided by their inherent capability of running algorithms in parallel. Combined with some freely available software solutions they tend to compete successfully with the commercial solutions. 


\title{
International Advanced Research Journal in Science, Engineering and Technology
}

\author{
Vol. 5, Issue 10, October 2018
}

\section{ACKNOWLEDGMENT}

This work is supported by contract No MEMF-151/08.03.2018, University of Mining and Geology “St. Ivan Rilsky”, Sofia, Bulgaria.

\section{REFERENCES}

[1]. International Energy Agency Executive Summary on Energy efficiency policy opportunities for electric motor-driven systems, OECD/IEA 2011

[2]. T. Javied, T. Rackow, R. Stankalla, J. FrankeJörg Franke, A Study on Electric Energy Consumption of Manufacturing Companies in the German Industry with the Focus on Electric Drives, Dec.2016, DOI 10.1016

[3]. B. Schlomann, T. Fleiter, A. Gerspacher, Datenbasis zur Bewertung von Energieeffizienzmaßnahmen 2008 (Auswertung für 2007), FraunhoferInstitut für System- und Innovationsforschung (FhG-ISI), Karlsruhe, Berlin, 2010

[4]. W. T. Thomson and R. J. Gilmore, "Motor current signature analysis to detect faults in induction motor drives. Fundamentals, data interpretation, and industrial case histories". Proceedings of the 32nd Turbomachinery Symposium . Texas A\&M University, 2003, pp.145-156

[5]. (2018) SEMAPI Test-I-Go Motor Current Signature Analysis, [Online]. Available: https://semapi.co.in/test-i-go/

[6]. (2018) MDA800A Motor Drive Analyzers, [Online]. Available: http://teledynelecroy.com/oscilloscope/mda800a-motor-drive-analyzer

[7]. IEEE Report, Report of Large Motor Reliability Survey of Industrial and Commercial Installations, Part I, IEEE Transactions on Industry Applications, Vol. IA-21, No. 4, 07/08 1985

[8]. Mohanty A., Machinery Condition Monitoring Principles And Practices, Indian Institute of Technology, CRC Press, ISBN-13: 978-1-46659305-3, 2015

[9]. F. Duan, Diagnostics of Rotor and Stator Problems in Industrial Induction Motors, Master's Thesis, Southwest Jiaotong University, China, 2005

[10]. G. Singh, S. Kazzaz, Induction Machine Drive Condition Monitoring and Diagnosis Research - A Survey, Electric Power Systems Research, vol. 64 , pp.145-158, 2003

[11]. Stopa M., B. De Jesus Cardoso Filho B., Load Torque Signature Analysis: an Alternative to MCSA to Detect Faults in Motor Driven Loads, IEEE Energy Conversion Congress and Exposition (ECCE), Raleigh, NC, USA, 2012, pp.4029-4036

[12]. Agoston, K. Vibration Detection of the Electrical Motors using Strain Gauges, 9th International Conference Interdisciplinarity in Engineering, INTER-ENG 2015, 8-9 October 2015, Tirgu-Mures, Romania, Procedia Technology 22 (2016), pp.767 - 772, doi:10.1016/j.protcy.2016.01.037

[13]. Irfan, M., Saad, N., Ibrahim, R., Asirvadam, V., Alwadie, A., Sheikh, M., An Assessment on the Non-Invasive Methods for Condition Monitoring of Induction Motors, Intech, Chapter 4, pp.87-129, Print ISBN 978-953-51-3203-5, DOI: 10.5772/67917, 2017

[14]. Merizalde Y., Hernández-Callejo L., Duque-Perez O., State of the Art and Trends in the Monitoring, Detection and Diagnosis of Failures in Electric Induction Motors, Energies 2017, 10, 1056; doi:10.3390/en10071056

[15]. D. Miljković, Brief Review of Motor Current Signature Analysis, HDKBR INFO Magazine, vol. 5, no. 1, pp. 14-26, [Online]. Available: https://hrcak.srce.hr/148715

[16]. C. Marcello, J. Fossatti, J. Terra, Fault diagnosis on induction motors based on FFT, in Dr. Salih Salih (ed.), Fourier Transform-Signal Processing, ISBN: 978-953-51-0453-7, InTech, 2012

[17]. Khadim Moin Siddiqui and V.K. Giri. "Broken Rotor Bar Fault Detection in Induction Motors using Wavelet Transform", Int. Conf Proc, IEEE, Computing, Electronics and Electrical Technologies [ICCEET], p. 1-6, Chennai, India, March, 2012

[18]. B. Bogert, M. Healy, J. Tukey, The Quefrency Alanysis of Time Series for Echoes: Cepstrum, Pseudo Autocovariance, CrossCepstrum and Saphe Cracking, Proceedings of the Symposium on Time Series Analysis (M. Rosenblatt, Ed) Chapter 15, pp. 209-243, New York, Wiley, 1963

[19]. Y. Hwang, K. Jen, Y. Shen, Application of cepstrum and neural network to bearing fault detection, Journal of Mechanical Science and Technology vol. 23, pp. 2730-2737, DOI 10.1007/s12206-009-0802-9, 2009, [Online]. Available: www.springerlink.com/content/1738-494x

[20]. Randall R., A History of Cepstrum Analysis and its Application to Mechanical Problems, Mechanical Systems and Signal Processing, ISSN 0888-3270, Vol. 97, 2017

[21]. A. Ourici, A. Ouari, Park's Vector Approach to detect an inter turn stator fault in a doubly fed induction machine by a neural network, Computer Science and Information Technology, DOI 10.5121/csit.2012.2503, 2012

[22]. S. Hanly, Accelerometers: Taking the Guesswork out of Accelerometer Selection, 2016, [Online]. Available: https://blog.mide.com/accelerometer-selection

[23]. Alorium Technology, 4205 Southtowne Dr. Eau Claire, WI 54701, [Online]. Available: https://www.aloriumtech.com

[24]. Website, [Online] Available: https://www.scilab.org

[25]. A. Quezada, Open Source FFT Spectrum Analyzer - An FFT spectrum analyser for machinery vibration analysis, using open source hardware and software, [Online]. Available: https://hackaday.io/project/12109-open-source-fft-spectrum-analyzer

[26]. S. Hanly, Vibration Analysis: FFT, PSD, and Spectrogram Basics, 2016, [Online]. Available: https://blog.mide.com/vibration-analysis-fft-psdand-spectrogram 